Gdańsk 2020, Nr. 43

https://doi.org/10.26881/sgg.2020.43.08

Alla Paslawska

Universität Lwiw / Аьвівський національний університет імені Івана Франка

ORCID-Nr: 0000-0002-7314-3171

\title{
Negationsforschung im Paradigmenwechsel um die Jahrtausendwende
}

Als 1989 die Germanistik an der Universität Gdansk gegründet wurde, stand das sowjetische Imperium direkt vor seinem Niedergang. Der Zerfall der Sowjetunion zeichnete sich unter anderem durch eine rasche Entwicklung nationaler Wissenschaften im postsowjetischen Raum, die sich in das gesamteuropäische Bildungssystem integrieren wollten. Dieser Prozess wurde auch durch einen Paradigmenwechsel in nationalen Linguistiken im Ganzen und in deren Germanistiken im Einzelnen begleitet. Das Ziel des Beitrags ist, am Beispiel einer universalen linguistischen Kategorie der Negation zu zeigen, welche wichtigen Schritte die Germanistik in den letzten 30 Jahren zurückgelegt hat. Die Untersuchung konzentriert sich auf die Inlandsgermanistik und zwei Auslandsgermanistiken - die polnische und die ukrainische.

Schlüsselwörter: Negation, Satznegation, Sondernegation, kontrastierende Negation, Fokus der Negation

Negation Research in the Paradigm Shift around the Turn of the Millennium. - When German studies was established at Gdansk University in 1989, the Soviet Empire was on the brink of its collapse. The collapse of the Soviet Union was characterized, among other things, by the rapid development of national sciences in the post-Soviet space, which wanted to integrate into the pan-European education system. This process was also accompanied by a paradigm change in national linguistics as a whole and in their German studies in particular. The aim of this article is to show, using the example of a universal linguistic category of negation, which important steps German studies has taken in the past 30 years. The study focuses on domestic and foreign German studies, namely in Poland and in Ukraine.

Keywords: Negation, Sentence Negation, Special Negation, Contrastive Negation, Focus of Negation.

Sprachliche Negation, um die es hier gehen wird, gehört neben Modalität, Temporalität und Aspekt zu den umstrittensten Phänomenen der Sprachwissenschaft. Alt wie die Sprachwissenschaft selbst, bleibt sie auch heute ein Prüfstein für moderne linguistische Theorien. An dieser Stelle sei eine Behauptung von Nussbaumer / Sitta (1986: 81) zitiert, welche auch heute aktuell bleibt:

„Die Negationsforschung ist in Bewegung. [...] Im Ganzen kann man sagen: Es hat einen beeindruckenden Sprung nach vorn gegeben - zu mehr Deutlichkeit, leider nicht zu mehr Einfachheit, im Gegenteil, und zu mehr Vollständigkeit“. 
Die in der Antike eingeleitete Diskussion zur Negation kommt in den 90er Jahren zu einer grundlegenden Unterscheidung zwischen zwei Forschungsparadigmen - dem „grammatischen“" und dem „pragmatischen“, wobei der semantischen Deutung der Negation (Jacobs 1982, Kürschner 1983) eine pragmatische gegenübergestellt wird, die Negation als Kategorie des kommunikativen Handelns betrachtet (Sennekamp 1979, Heinemann 1983). Innerhalb der grammatischen Richtung bildet sich jedoch auch eine klare Grenze zwischen den traditionell und transformationell ausgerichteten Grammatiken. Als traditionell werden hier Grammatiken verstanden, die den transformationellen Ansätzen der 70er Jahre vorangehen und/oder solche, die ihnen gegenübergestellt werden und die eher praktisch konzipiert sind.

Bei der Behandlung der Negation betrifft diese Einteilung in erster Linie die Unterscheidung der traditionellen Grammatik zwischen Satz- und Sondernegation (Helbig/ Albrecht 1993) als zwei grundsätzlich unterschiedlichen Arten der Negation und den Verzicht darauf der transformationellen Grammatik, deren ersten Ansätze in diesem Format auf den Aufsatz von W. Hartung (1971) und "Studien zur Negation im Deutschen“ von G. Stickel (1970) zurückgehen. Im Unterschied zur traditionellen Grammatik behaupten beide Autoren, dass eine Unterscheidung zwischen zwei Arten der Negation keinen Sinn hat und dass sich die Sondernegation auf die Satznegation zurückführen lässt. Gerade in den 90er Jahren erlebt die Untersuchung der Negation ihre Blütezeit. Denn auf diese Zeit fällt die Entwicklung einer Reihe von innovativen Forschungsbereichen der sogenannten Negationslinguistik.

Der vorliegende Beitrag wird im ersten Teil den Stand der Negationsforschung der 90er Jahre zusammenfassen. Im zweiten Teil soll ein Überblick über die Ansätze zur Negation der letzten dreißig Jahre verschafft werden. Ein Ausblick in die Negationsforschung wird im dritten Teil des Beitrags präsentiert.

Mitte der 90er Jahre bestehen in der Negationsforschung kontroverse Meinungen bezüglich der Natur der Negation und ihrer semantischen Arten. Dabei lassen sich psychologische, modale, „objektive“ und formal-logische Auffassungen der Negation unterscheiden.

Der Psychologismus bringt den Ursprung der Negation mit dem Ausdruck der primitiven Interjektion der Abneigung in Zusammenhang. Das soll erklären, warum in vielen Sprachen die Negation mit einem Nasallaut anfängt. Obwohl diese Theorie, die wohl auf Otto Jespersen (1917) zurückgeht, inzwischen als überholt gilt, wird später jedoch gezeigt, dass man sie zu früh aufgegeben hat und dass sie moderne Theorien zum Negationserwerb bei Kindern untermauern kann. Mit Hilfe der psychologischen Begriffe des Glaubens und Nichtglaubens charakterisierte Äußerungen Bertrand Russell. Nach seiner Meinung drücken die Sätze mit Negationswörtern nicht nur einen bestimmten Inhalt aus, sondern auch das Verhältnis des Sprechers zum konkreten Inhalt. Jeder Glaube korreliert mit dem Nichtglauben, der auch wahr oder falsch sein kann. „Wenn ich an ,nicht p' glaube, dann glaube ich nicht an ,p' (Russell 1957: 162).

Zahlreiche Linguisten schreiben der Negation die Bedeutung der sogenannten objektiven oder subjektiven Modalität zu. Als Kategorie, die die objektive Realität hinsichtlich der Nichtrealität der Fakten der Wirklichkeit widerspiegelt, wird die Negation der Affirmation als objektiver Modalität der Wirklichkeit gegenübergestellt. Die subjektive Modalität der 
Negation wird in der Bewertung der Realität/Nichtrealität des Gesagten durch den Sprecher gesehen (Vinogradov 1947, Peschkovskij 1957, Admoni 1986). Solche Ansichten sind auf eine zu breite Auffassung der Modalität zurückzuführen. Negierte Sätze widerspiegeln die Wirklichkeit genau wie affirmative, wenn sie wahr sind. Ist der Satz falsch, dann ist es egal, ob er negativ oder affirmativ ist - er widerspiegelt den realen Sachverhalt nicht. Aber unbestritten bleiben enge Zusammenhänge und Überschneidungen zwischen der Negation und Modalität. Die sowjetische Linguistik sah oft in der Negation eine ontologische Kategorie, deren Referent objektive „Uneinigkeit“ (razjedinennost') eines Gegenstandes und seiner Merkmale oder die Abwesenheit objektiver Verbindungen zwischen Begriffen ausdrückt (Vasiljeva 1958: 149). Diese hypermaterialistischen Annahmen, die übrigens bereits in der altindischen Philosophie vertreten waren, wurden in postsowjetischen Untersuchungen zur Negation verworfen. Denn es gibt keine negative Realität, genau wie die Gegenstände ohne ihre Merkmale nicht existieren können.

Formal-logische Definitionen der Negation haben wesentlich deren Auffassung in der Sprachwissenschaft beeinflusst. Die Fähigkeit negativer Sätze, die Wirklichkeit wiederzugeben, wurde auf ihre Wahrheit zurückgeführt (vgl. Frege 1918/1919, Russell 1905). Etwas anders als in der Logik wurde jedoch traditionelle Unterscheidung zwischen den Arten der Negation getroffen. Während klassische Logik von zwei Klassen der Negation ausgeht, der Satz- und der Prädikatsnegation, unterscheidet die traditionelle Grammatik zwischen der Satzund Sondernegation. Im Folgenden soll auf Probleme dieser Einteilung eingegangen werden.

Die übliche Unterscheidung herkömmlicher Grammatiken zwischen zwei Arten der Negation beruht darauf, dass bei der Satznegation ein ganzer Satz, bei der Sondernegation nur ein Teil davon der Negation unterliegt:

(1) Hilla hat auf Andreas nicht gewartet. (Satznegation)

(2) Nicht auf Andreas hat Hilla gewartet. (kontrastierende Negation)

Im Beispiel (1) ist der Sachverhalt negiert, dass Hilla auf Andreas gewartet hat. Das Beispiel (2) besagt, dass Hilla auf eine andere Person als Andreas gewartet hat. Beide Arten der Negation unterscheiden sich auch dadurch, dass nur bei der Sondernegation die Existenz eines Sachverhalts behauptet werden kann. Nur aus dem zweiten Satz lässt sich schließen, dass es einen Sachverhalt gegeben hat, in dem Hilla auf jemand gewartet hat. Normalerweise wird die kontrastierende Negation durch ihre Position unmittelbar links vom Satzglied, das negiert wird, markiert. Der Negation kann dabei jedes Satzglied unterliegen.

(3) Nicht Hilla hat heute die Tasche gekauft.

(4) Hilla hat nicht heute die Tasche gekauft.

(5) Hilla hat heute nicht die Tasche gekauft.

(6) Hilla hat heute die Tasche nicht gekauft.

Bei Bedarf kann kontrastierende Negation mittels der sondern- oder aber-Konstruktionen identifiziert werden.

(7) Nicht Hilla hat heute die Tasche gekauft, sondern Andreas. 
Eine Mehrdeutigkeit zwischen zwei Arten der Negation kann bei der Negation des Prädikats (vgl. 6) ausgelöst werden: der Satz kann zwei Lesarten - mit einer Satz- oder einer kontrastierenden Negation haben. Bei neutraler Satzintonation mit der Akzentuierung der Partikel nicht wird die Satznegation ausgelöst. Kontrastierende Hervorhebung des Prädikats führt zur kontrastierenden Negation im Satz.

(8) Hilla hat heute die Tasche NICHT gekauft. (Satznegation)

(9) Hilla hat heute die Tasche nicht GEKAUFT (sondern ausgeliehen). (kontrastierende Negation)

In einem negierten Satz kann intonatorische Hervorhebung einer beliebigen Konstituente deren kontrastierende Negation auslösen.

(10) Hilla hat heute die Tasche NICHT gekauft (aber Andreas).

(11) Hilla hat HEUTE die Tasche NICHT gekauft (aber gestern schon).

(12) Hilla hat heute die TASCHE NICHT gekauft (aber ein Buch).

(13) Hilla hat heute die Tasche nicht GEKAUFT (sondern ausgeliehen).

Damit kann die traditionell verbreitete These darüber widerlegt werden, dass kontrastierende Negation immer links von der negierten Konstituente steht.

Der Wirkung der Negation können Satz- und Wortbestandteile von unterschiedlicher Komplexität unterliegen. Das kann eine Wortgruppe (14), ein Wort (15) oder sogar ein Präfix (16) sein.

(14) Hilla ist heute nicht zur Arbeit gegangen (sondern nach Wien gefahren).

(15) Hilla hat nicht Andreas eingeladen (sondern Alla).

(16) Hilla hat die Flasche nicht VERkorkt (sondern ENTkorkt).

Neuere Ansätze gehen von der Annahme aus, dass der Negation als einstellige Wahrheitswertfunktion immer ein logischer Satz unterliegt, d. h. das Prädikat und seine Argumente. Daher hat die Negation des Prädikats allein oder eines einzelnen Arguments keinen Sinn (Jacobs 1991, Dahl 1993: 915, Zifonun 1997: 846). Der Wirkungsbereich der Negation ergibt sich aus dem Zusammenspiel solcher Komponenten, wie Wortfolge, Intonation und Kontext des Negationsträgers. Gerade die intonatorische Hervorhebung (Fokussierung und Kontrastierung) der Negation können den Effekt der Sondernegation verursachen und den Eindruck verschaffen, als wäre nicht der ganze Satz von der Negation betroffen, sondern nur ein Teil davon. Eine korrekte Analyse der Negation muss das berücksichtigen und zwischen mindestens drei Arten der Negation unterscheiden: 1) der neutralen (pauschalen) Negation, 2) der fokusbezogenen Negation und 3) der kontrastierenden Negation (vgl. Zifonun 1997: 854).

Bei der neutralen Negation wird ein Sachverhalt in Abrede gestellt, ohne zu differenzieren, welchen Satzteil die Negation betrifft.

(17) Andreas beschwert sich nicht über das Wetter.

Syntaktisch lässt sich eine solche Negation durch eine Reihe von Merkmalen beschreiben: Stabilität der Wortfolge, neutrale Intonation, Mittelfeldposition im Satz mit Verbzweitstellung. 
Der fokusbezogenen Negation liegt die neutrale Negation zugrunde. Zusätzlich wird jedoch mit Hilfe entsprechender Akzentuierung und eventuell Topikalisierung eine Satzkonstituente besonders hervorgehoben.

(17a) Andreas beschwert sich nicht über das Wetter.

(17b) ÜBER DAS WETTER beschwert sich Andreas nicht.

Es bleibt dabei offen, ob in (17a) eine andere Person als Andreas sich über das Wetter beschwert oder ob in (17b) sich Andreas über jemanden oder über etwas anderes als das Wetter beschwert.

Für kontrastierende Negation sind die Merkmale sowohl der neutralen als auch der fokusbezogenen Negation eigen, aber darüber hinaus eröffnet sie eine Menge von Alternativen für den fokussierten Satzteil, die bei Bedarf durch die sondern- oder aber-Konstruktionen expliziert werden können:

(17c) Nicht Andreas beschwert sich über das Wetter (sondern Hilla).

(17d) Andreas beschwert sich nicht über das Wetter (sondern über die Politik).

Im Unterschied zu den beiden anderen Negationsarten wird nur bei der kontrastierenden Negation die Existenz des Sachverhalts nicht in Zweifel gezogen. Der Negationsträger steht in diesem Fall vor dem kontrastierten Satzteil. Wie man sieht, ist beim Auslösen der kontrastierenden Negation ebenfalls das Zusammenspiel von dem semantischen Wirkungsbereich, der syntaktischen Position und der Akzentuierung relevant.

Eine weitere wichtige Erkenntnis im Bereich der Negation der letzten dreißig Jahre war eine klare Unterscheidung zwischen Negation und Negierung (Engel 1996, Heinemann 1983, Schmidt 1973). Negierung lässt sich aus einem Bedürfnis begründen, Widerspruch, Absage, Ablehnung oder Verbot zum Ausdruck zu bringen. Damit ist eine Reaktion auf eine explizite oder implizite Vorgängeräußerung gemeint. Von der Negation kann sie dadurch unterschieden werden, dass Negation als eine Operation auf sprachlichen Inhalten anzusehen ist, während Negierung eine Handlung ist, die man vollzieht, indem man Ausdrücke einer Sprache verwendet (Jacobs 1991: 561). Dabei betrifft Negierung nicht direkt eine reale (mögliche) Welt, sondern sie bezieht sich vielmehr auf Behauptungen, Annahmen oder Erwartungen eines Sprechers über Sachverhalte der Welt (vgl. Schmidt 1973), ohne dabei an bestimmte morphologische Mittel gebunden zu sein.

$\mathrm{Zu}$ negierenden Sprechhandlungen werden (mit einigen Modifikationen) Zurückweisen, Bestreiten und Verbieten gezählt (vgl. Blühdorn 2012, Engel 1996, Heinemann 1983, Schmidt 1973). Unter Zurückweisen versteht man eine negierende Sprechhandlung, die eine vorangehende Aufforderung voraussetzt, die vom Sprecher als nicht akzeptabel abgelehnt wird:

(18) [ - Trinken Sie den Wein aus! $]$

(19) - Es ist nicht erlaubt im Dienst.

Die zahlreichen Arten des Zurückweisens kann man als “jemand will nicht, dass p" paraphrasieren.

Widersprechen ist eine negierende Sprechhandlung, durch die der Sprecher die Gültigkeit einer Aussage bestreitet: 
(20) [ - Es regnet nicht.]

(21) - Doch! Es regnet.

Widersprechen lässt sich durch "es ist nicht so, dass p"umschreiben.

$\mathrm{Zu}$ Verbieten gehören Sprechhandlung, die das Ziel verfolgt, weitere Handlungen des Kommunikationspartners zu blockieren.

(22) [ - Ich möchte ein Eis!]

(23) - Du darfst kein Eis essen.

Paraphrasieren kann man Verbieten durch "es ist nicht erlaubt, dass p".

Die Forschung zum Zusammenspiel der Negation mit der Syntax, Semantik, Pragmatik und Prosodie des Satzes hat zu einer weiteren wichtigen Unterscheidung zwischen entsprechenden Bereichen der Negation geführt. So wird in Jacobs (1991) zwischen folgenden Negationsbereichen unterschieden:

(24) Negationsbereiche:
a. Syntaktischer Bereich von Neg
b. Semantischer Bereich von $\mathrm{Neg}$
c. Intonatorischer Bereich von $\mathrm{Neg}$
d. Pragmatischer Bereich von Neg

Als syntaktischer Bereich von Neg wird in einem Satz S derjenige Teil des Satzes betrachtet, der nach Aufweis der Konstituentenstruktur von $S$ vom ersten verzweigenden Knoten dominiert wird, der auch $\mathrm{Neg}$ dominiert. In einem universalen Satzmodell nach Pollock (1989: 397) wird der Negation eine Position zwischen zwei funktionalen Projektionen des Satzes zugewiesen - unter der Tempusphrase und über der Agreementobjektphrase:

(25)

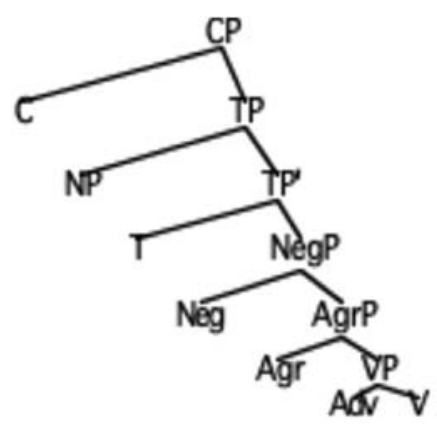

Mechanismen der Ableitung der Oberflächenstruktur lassen sich am nächsten Beispiel veranschaulichen: 
(26)

\section{Es stört mich nicht.}

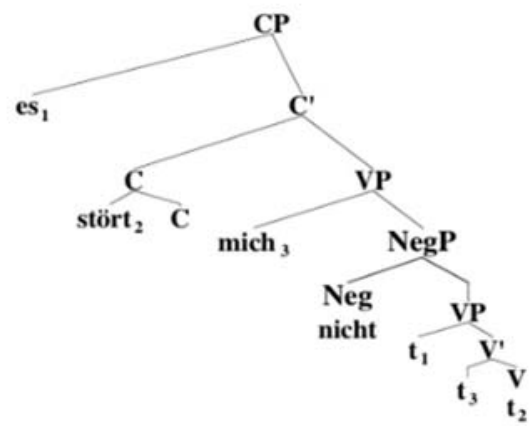

Das Verb stören wird samt seinen Argumenten es und mich in der Prädikatsphrase VP generiert, die Negation - darüber. Das Verb bewegt sich dann in eine freie Position und zwar zum Complementierer $C$, indem es die Spur $t_{2}$ hinterlässt. Das Subjekt bewegt sich in das Vorfeld und hinterlässt seine Spur $t_{1}$ in der Basisposition. Das direkte Objekt mit der Spur $t_{3}$ wird an die VP adjungiert. Die Negation bleibt hier dagegen in ihrer ursprünglichen Position.

Als semantischer Bereich der Negation wird der Teil der semantischen Repräsentation des Satzes definiert, auf den sich die Wirkung der Negation ausbreitet, d. h. das Prädikat mit seinen Argumenten und gelegentlich Cirkumstanten. Die letzten können sowohl im Bereich der Negation als auch außerhalb ihrer Wirkung stehen:

(27a) Man will absichtlich nicht verstanden werden (außerhalb des Wirkungsbereichs der Negation)

(27b) [absichtlich [man will [NEG [verstanden werden]]]].

(28a) Ich werde nicht absichtlich hyperaktiv (im Wirkungsbereich der Negation).

(28b) [NEG [absichtlich [ich werde hyperaktiv]]].

Unter dem Intonationsbereich der Negation versteht man einen Teil des Satzes, der durch eine Hervorhebung die Negation des hervorgehobenen Materials bewirkt und dafür eine Klasse von Alternativen eröffnet:

(29) Hilla geht nicht zur Uni.

Je nachdem, welcher Satzteil fokussiert wird, werden unterschiedliche Klassen von Alternativen eröffnet: HILLA geht nicht zur Uni $\Rightarrow$ \{Andreas, Hans, Maria...\} geht zur Uni; Hilla GEHT nicht zur Uni $\Rightarrow$ Sie $\{$ fährt, fliegt, rennt... $\}$ zur Uni; Hilla geht nicht zur UNI $\Rightarrow$ Sie geht \{ins Kino, nach Hause, zu ihrer Freundin...\}.

Als pragmatischer Bereich des Satzes werden diejenigen Implikate des Satzinhalts verstanden, die durch Negation betroffen werden. Der pragmatische Bereich der Negation ist kontextabhängig und kann nur dadurch bestimmt werden (Jacobs 1991: 574):

(30) - Wohnt Hillas Schwester bei ihrer Tante? - Nein, Hillas Schwester wohnt nicht bei ihrer Tante, 

a) denn sie hat eine eigene Wohnung.
b) denn sie hat überhaupt keinen festen Platz.
c) denn Hilla hat gar keine Schwester.

Je nach dem Kontext kann negiert werden, dass a) Hillas Schwester in der Wohnung einer anderen Person wohnt, b) dass sie überhaupt irgendwo wohnt, c) dass sie existiert.

In polnischen Grammatiken wird der Negation nicht viel Aufmerksamkeit gewidmet: "Traditional Polish grammars, e.g., Krasnowolski (1911), Szober (1953) and Klemensiewicz (1968), do not mention negation at all, while many other grammar textbooks do not deal with the nature of negation per se." Demgegenüber wird in den 90er Jahren die Negation zu einem viel diskutierten Thema (Greszczuk (1993), Kupść / Przepiórkowski (1998), Blaszczak (1998)). Mittels der Negation werden andere Theorien überprüft, so wie das z. B. Gliwiński (1982) macht, indem er in einer deutsch-polnischen kontrastiven Analyse mittels der Partikel nie den grammatischen Status des Kopulaverbs und die damit verbundenen, durch die Negation ausgelösten Transformationen in entsprechenden Kopulasätzen diskutiert.

Sehr umstritten ist die Frage nach dem morphologischen Status von nie im Polnischen. So vertreten Śpiewak / Szymańska (1995), Witkoś (1996) die Ansicht, dass nie ein Klitikon ist. Saloni / Świdziński (1985) sind jedoch der Meinung, dass es über den morphologischen Status verfügt, während Świdziński (1992) dazu neigt, verbale Negation als rein syntaktische Erscheinung zu deuten: nie ist eine morphologische Einheit, die über syntaktische Regeln an das Verb angefügt wird. Kupść / Przepiórkowski (1998) sehen in der verbalen Negation im Polnischen nie ein Präfix, das durch syntaktische Regeln ans Verb angefügt wird.

Von besonderem Interesse ist die für das Polnische vorgeschlagene Analyse von negativen Indefinita von Błaszczak (1998), in der sie zeigt, dass sogenannte $n$-Wörter nicht als Negative Quantoren interpretiert werden sollen, sondern vielmehr als spezielle Negative Polaritätselemente (NPIs), die eine positive Bedeutung haben, aber im Skopus einer Negation stehen müssen. Eine ähnliche Analyse wird für das Ukrainische (Paslawska 2001) vorgeschlagen, wo $n$-Wörter als NPIs gedeutet werden, deren Negation nur ein morphologischer Reflex einer an einer anderen Stelle präsenten Negation ist.

Weitere Richtungen der ukrainischen Negationsforschung sind kognitivistische Ansätze, die Negation als eine der Möglichkeiten der Konzeptualisierung der Wirklichkeit betrachten, die die im Bewusstsein des Adressaten vorhandenen zwischenreferentiellen Verhältnisse verändern (Kryschtaljuk 2009). Man versucht für einzelne Negierungsarten entsprechende Framemodelle zu entwickeln (Krajnyk 2016).

Eine andere Entwicklung der Negationsforschung bildet die Untersuchung des Negationserwerbs bei Kindern (Paslawska 2009), wobei eine Systematik des zyklischen Erwerbs einzelner Negationen festgestellt wird, die es ermöglicht, bestimmte Parallelen zur Evolution der Sprache im Allgemeinen aufzustellen. Die Idee einer zyklischen Entwicklung der Negation geht, wie bekannt, auf Otto Jespersen (1917) zurück. Die Ergebnisse einer solchen Forschung mit dem Heranziehen mehrerer Sprachen könnten sowohl den Spracherwerb im Ganzen als auch die Mechanismen der Bildung von syntaktischen Strukturen besser zu verstehen helfen. 
Zahlreich sind die Aufsätze zu Rolle und Funktionen der Negation in der Interkulturellen Kommunikation, die sich mit Mono- und Polynegation, non- und paraverbalen Negationsmitteln beschäftigen, sowie didaktisch-methodische Beiträge, die sich den Besonderheiten der Negation in jeder der behandelten Sprachen widmen. Es ist bekannt, dass die Unkenntnis von wichtigen nonverbalen Kommunikationsmitteln zu schweren Verletzungen der Kommunikation führen kann. Man denke, z. B. an zahlreiche nonverbale Negationsmittel - Gestik (Kopfschütteln), Mimik (Naserümpfen) sowie paraverbale - die Möglichkeit durch Stimmhöhe, spezielle Intonationskurven, Sprechgeschwindigkeit, durch ein Zittern oder Lachen in der Stimme (z. B. das sarkastische $\mathrm{Ha}-\mathrm{h} a$ ) die Negation zum Ausdruck zu bringen. Diese Mittel sind jedoch kulturspezifisch und können manchmal das entgegengesetzte bedeuten (z. B. Kopfnicken für "Nein" im Bulgarischen).

Zusammenfassend lässt sich sagen, dass man am Beispiel der Negation eine dynamische Entwicklung alter und neuer linguistischer Paradigmen um die Jahrtausendwende beobachten kann. Positiv ist dabei die Tatsache, dass neuere Ansätze auf der Tradition aufbauen und dass die Inlandsgermanistik dabei mit den Auslandsgermanistiken (hier der polnischen und ukrainischen) kooperiert und alle einander bereichern.

\section{Literatur}

Admoni, Vladimir (1986): Teoretitscheskaja grammatika nemeckogo jazyka [Theoretische Grammatik der deutschen Sprache]. Moskva: Prosveschtschenije.

Błaszczak, Joanna (1998): Towards a Binding Analysis of Negative Polarity Items. In: Linguistics in Potsdam 4, 1-37.

Blühdorn, Hardarik (2012): Negation im Deutschen: Syntax, Informationsstruktur, Semantik. Tübingen: Narr.

Dahl, Östen (1979): Typology of sentence negation. In: Linguistics 17/1-2, 79-106.

Engel, Ulrich (1988): Deutsche Grammatik. Heidelberg: Julius Groos Verlag.

Frege, Gottlob (1918-1919): Die Verneinung. In: Beiträge zur Philosophie des deutschen Idealismus (I), 143-157.

Gliwiński, Tomasz (1982): Zur Negation im Kopulasatz im Deutschen und Polnischen. In: Acta Universitatis Lodziensis. Folia Linguistica 3, 43-51.

Greszczuk, Barbara (1993): Sktadniowe wyktadniki negacji i ich funkcje w historii jezyka polskiego [Syntaktische Exponenten der Negation und deren Funktion in Geschichte des Polnischen]. Rzeszów: Wydawnictwo WSP.

Hartung, Wolfdietrich (1964): Die Negation in der deutschen Gegenwartssprache. In: Deutsch als Fremdsprache 2, 13-19.

Heinemann, Wolfgang (1983): Negation und Negierung. Handlungstheoretische Aspekte einer linguistischen Kategorie. Leipzig: Enzyklopädie.

Helbig, Gerhard/ Albrecht Helga (1993): Die Negation. 6., durchgesehene Aufl. Leipzig [etc.]: Langenscheidt.

Jacobs, Joachim (1982): Syntax und Semantik der Negation im Deutschen. München: Fink. 
Jacobs, Joachim (1991): Negation. In: Arnim von Stechow, Dieter Wunderlich (Hg.): Semantics: An International Handbook of Contemporary Research. Berlin, New York: de Gruyter, 560-596.

Jespersen, Otto (1917): Negation in English and other Languages. Köbenhavn: Kgl. Danske Videnskabernes Selskab, Historisk-Filologiske Meddelelser 1 (5). [Reprinted in: Selected writings of Otto Jespersen. - London: Allen and Unwin, no year.].

Klemensiewicz, Zenon (1968): Podstawowe wiadomości z gramatyki języka polskiego. [Grundkenntnisse der polnischen Grammatik]. Warszawa: PWN.

Krasnowolski, Antoni (1911): Gtówne zasady sktadni polskiej [Grundprinzipien der Syntax des Polnischen]. Warszawa: Wydawnictwo M. Arcta.

Kraynyk, Olha (2016): Die negierenden Sprechhandlungen. In: Science and Education a New Dimension: Philology. Budapest. 99, 19-22.

Kryschtaljuk, Hanna (2009): Zaperetschennja v sutschasnomu amerykans'komu dyskursi: funkcional'nyj ta linhwokohnityvnyj aspekty [Die Negation im modernen amerikanischen Discourse: funktionaler und linguo-kognitiver Aspekte]. Diss. Donezk: Donezkyj universytet.

Kupść, Anna / Przepiórkowski, Adam (1997): Morphological Aspects of Verbal Negation in Polish. In: Peter Kosta / Jens Frasek (Ed.): Current Approaches to Formal Slavic Linguistics. Frankfurt/M.: Lang, 337-346,

Kürschner, Wilfried (1983): Studien zur Negation im Deutschen (= Studien zur deutschen Grammatik 12). Tübingen: Narr.

Nussbaumer, Markus / Sitta, Horst (1986): Negationstypen im Spannungsfeld von Satz- und Sondernegation. In: Deutsch als Fremdsprache 23, 58-84.

Paslawska, Alla (2011): Negative Polaritätselemente und ihre Lizensierung im Ukrainischen. In: Proceedings of Formal Description of Slavic Languages 4. Potsdam: University Potsdam, 116-118.

Paslawska, Alla (2009): Zaperetschennja v mownomu ontohenezi [Die Negation im Spracherwerb]. Kirowohrad: Naukovi zapysky Kirovohrads'koho universytetu imeni Wolodymyra Wynnytschenka $81 / 2,227-230$.

Peschkovskij, Aleksandr (1956): Russkij sintaksis v nautschnom osweschtschenii. [Die russische Syntax aus wissenschaftlicher Sicht]. Moskva: Utschpedgiz.

Pollock, Jean-Yves (1989): Verb Movement, Universal Grammar, and the Structure of IP. In: Linguistic Inquiry 20, 365-424.

Przepiórkowski, Adam / Kupść Anna (1997). Verbal negation and complex predicate formation in Polish. In: Texas Linguistic Forum 38, Proceedings of the 1997 Texas Linguistic Society Conference. Texas: Austin, 247-261.

Russell, Bertrand (1905): On Denoting. In: Mind 14, 479-493.

Russell, Bertrand (1957): Tschelowetscheskoe poznanie, ego sfera i granizy [Das menschliche Wissen]. Moskva: Izdatel'stvo inostrannoj literatury.

Saloni, Zygmunt / Świdziński, Marek (1985): Sktadnia wspótczesnego języka polskiego (wyd. 2. zmienione) [Syntax des Gegenwartspolnischen (2. veränderte Aufl.)]. Warszawa: PWN.

Schmidt, Siegfried J. (1973): Texttheoretische Aspekte der Negation. In: Zeitschrift für germanistische Linguistik 1, 178-208.

Sennekamp, Marita (1979): Die Verwendungsmöglichkeiten von Negationszeichen in Dialogen. München: Hueber. 
Śpiewak, Grzegorz / Szymańska, Izabela. (1995): A Poll on Pollock - Functional Categories in Polish. In: Gussmann, Edmund (ed.): Licensing in Syntax and Philology. Lublin: Wydawnictwo Folium, 125-145.

Stickel, Gerhard (1970): Untersuchungen zur Negation im heutigen Deutsch. Braunschweig: Vieweg. Świdziński, Marek (1992): Gramatyka formalna jezyka polskiego [Formale Grammatik des Polnischen]. Warszawa: Wydawnictwo Uniwersytetu Warszawskiego.

Szober, Stanisław (1953): Gramatyka jezyka polskiego [Grammatik des Polnischen]. Warszawa: Nasza Księgarnia.

Vasiljeva, Svetlana (1958): K voprosu o prirode otricanija [Zur Frage der Natur der Negation] In: Sbornik rabot Leningradskogo technologitscheskogo instituta pischtschevoj promyschlennosti, 137-157.

Vinogradov, Viktor (1947): Russkij jazyk. Grammatitscheskoje utschenije o slove [Russische Sprache. Grammatisches Studium über das Wort]. Moskva / Leningrad: Utschpedgiz.

Witkoś, Jacek (1996): On NegP and structure of the Polish clause. In: Papers and Studies in Contrastive Linguistics XXX, 67-96.

Zifonun, Gisela / Hoffmann, Ludger / Strecker, Bruno et al. (1997): Grammatik der deutschen Sprache. Bd. 1. Berlin [etc.]: de Gruyter. 